University of Nebraska - Lincoln

DigitalCommons@University of Nebraska - Lincoln

Alexei Gruverman Publications

Research Papers in Physics and Astronomy

December 2006

\title{
Thickness profiles through fatigued bulk ceramic lead zirconate titanate
}

Nina Balke

Institute of Materials Science, Darmstadt University of Technology, Petersenstrasse 23, Germany, balken@ornl.gov

Doru C. Lupascu

Institute of Materials Science, Dresden University of Technology, 01062 Dresden, Germany, doru.lupascu@uni-due.de

Thomas Blair

North Carolina State University, Raleigh

Follow this and additional works at: https://digitalcommons.unl.edu/physicsgruverman

Part of the Physics Commons

Balke, Nina; Lupascu, Doru C.; and Blair, Thomas, "Thickness profiles through fatigued bulk ceramic lead zirconate titanate" (2006). Alexei Gruverman Publications. 31.

https://digitalcommons.unl.edu/physicsgruverman/31

This Article is brought to you for free and open access by the Research Papers in Physics and Astronomy at DigitalCommons@University of Nebraska - Lincoln. It has been accepted for inclusion in Alexei Gruverman Publications by an authorized administrator of DigitalCommons@University of Nebraska - Lincoln. 


\title{
Thickness profiles through fatigued bulk ceramic lead zirconate titanate
}

\author{
Nina Balke \\ Institute of Materials Science, Darmstadt University of Technology, Petersenstrasse 23, \\ 64287 Darmstadt, Germany \\ Doru C. Lupascua) \\ Institute of Materials Science, Dresden University of Technology, 01062 Dresden, Germany \\ Thomas Blair and Alexei Gruverman \\ Department of Materials Science and Engineering, North Carolina State University, Campus Box 7920, \\ Raleigh, North Carolina 27695
}

(Received 19 June 2006; accepted 10 September 2006; published online 15 December 2006)

\begin{abstract}
Wedge-cut samples of fatigued ferroelectric lead zirconate titanate ceramics were investigated using piezoresponse force microscopy in conjunction with conventional electrical hysteresis measurements. The local clamping of domains is monitored at different depths in the sample. The coercive fields in grains near the electrodes differ for different materials and preparation methods of the electrodes. For silver, fatigue consistently generates a space charge in the depth of the sample. For platinum electrodes, the fatigue behavior scatters strongly. Microscopically, it either occurs directly underneath the electrodes or resembles the behavior of the silver electrodes in other samples. (C) 2006 American Institute of Physics. [DOI: 10.1063/1.2395600]
\end{abstract}

\section{INTRODUCTION}

Fatigue in ferroelectric materials has been a continuous concern in actuators ${ }^{1-7}$ as well as thin film devices. ${ }^{8-20}$ Due to the large market shares anticipated in fuel injection systems and continuous development of ferroelectric memories, a good understanding of the underlying effects remains an important issue to be solved as underlined by the large number of publications already devoted to this subject. Overall, fatigue yields a loss of switchable polarization and certain offsets irrespective of device geometry. ${ }^{4,16}$ It depends on the loading conditions and the particular ferroelectric and electrode materials whether offsets ${ }^{2,4,7,21}$ or the loss of switchable polarization $^{1-19}$ is more pronounced. The former is most common for unipolar loading in actuators, the latter in bipolar fatigued films. ${ }^{16}$ Often both effects arise simultaneously to different extent. Potential mechanisms that have been considered during the last two decades are microcracking, ${ }^{2,22}$ oxygen vacancy clustering at electrodes, ${ }^{23}$ defect cluster growth, ${ }^{24}$ suppression of domain nucleation at the electrode interface, ${ }^{25}$ charge carrier drift, ${ }^{26-28}$ breakdown, ${ }^{28}$ charge carrier injection, ${ }^{17,29}$ depletion regions in the electrodes, ${ }^{30}$ injection of protons during processing, ${ }^{31}$ ionization of foreign dopants, ${ }^{32}$ or ionization even of the host ions. ${ }^{14}$ The role of grain boundaries is yet unclear. ${ }^{33-35}$

The dominant role of the electrode interface is by now well established for thin films under bipolar loading as used in memory applications. ${ }^{36-38}$ While ferroelectrics of the bismuth-layered perovskite family of compounds seem to withstand fatigue irrespective of the electrode chosen, ${ }^{39,40}$ actuators still have to rely on the lead zirconate titanate (PZT) system due to the negligibly low strains provided by the bismuth-layered compounds. PZT shows offsets and

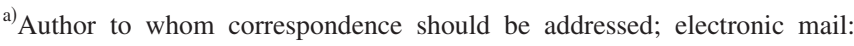
doru.lupascu@tu-dresden.de
}

strongly reduced switching under bipolar loading and offsets due to unipolar or sesquipolar fatigue., ${ }^{2,7,41,42}$ Oxide electrodes of $\mathrm{RuO}_{2-x}, \mathrm{YBa}_{2} \mathrm{Cu}_{3} \mathrm{O}_{7}$, or $\mathrm{IrO}_{x}$ (Refs. 43-45) have proven to sufficiently reduce the fatigue effects in PZT thin films under bipolar loading so that memory applications have become feasible for bipolar cycling exceeding $10^{11}$ cycles. $^{46,47}$ Commercial versions of ferroelectric memory devices show sufficient fatigue resistance even for metallic platinum electrodes if iridium oxide is used as a buffer. ${ }^{48}$ Despite the many different approaches to explaining the fatigue effect in ferroelectrics, it is still not fully clear which microscopic mechanisms are responsible for the deficiencies of metallic electrodes nor why the improvements have become possible for the Pt-PZT combination of electrode and ferroelectric incorporating Ir.

The present publication is devoted to the study of the near electrode region of bulk ferroelectrics with metal electrodes subject to bipolar fatigue loading. It is intended to improve the understanding of the switching dynamics of the domains in bulk lead zirconate titanate on the microscopic level by utilizing piezoresponse force microscopy (PFM) as an imaging technique. The advantage of using bulk samples resides in the fact that electrodes can be applied in a nonreducing environment which is otherwise common practice for the application of $\mathrm{Pt}$ electrodes to thin films and may be responsible for some of the difficulties encountered in thin film fatigue due to protons. Bulk switching is displayed for comparison. $^{49}$

\section{EXPERIMENTAL PROCEDURE}

All measurements were performed on commercial PZT samples (PIC 151) which were obtained from PI Ceramics (Lederhose, Germany). The composition is approximately 


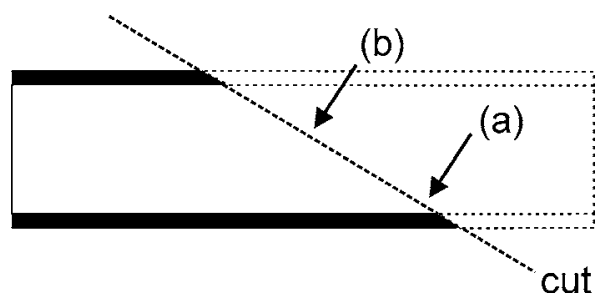

FIG. 1. Illustration of the wedge shaped samples cut along the dashed line at an angle of $10^{\circ}$. PFM measurements were performed (a) near the electrode or (b) deep in the bulk.

$\mathrm{Pb}_{0.99}\left[\mathrm{Zr}_{0.45} \mathrm{Ti}_{0.47}\left(\mathrm{Ni}_{0.33} \mathrm{Sb}_{0.67}\right)_{0.08}\right] \mathrm{O}_{3}$. The samples were disk shaped with a diameter of $10 \mathrm{~mm}$ and a thickness of $1 \mathrm{~mm}$. The average grain size was about $6 \mu \mathrm{m}$.

\section{A. Fatigue procedure}

The samples were polished using diamond pastes of 15 , 6 , and $3 \mu \mathrm{m}$ down to $1 \mu \mathrm{m}$ grid size utilizing an alcohol based lubricating fluid (DP-Lubricant Blue®) before the electrodes were sputtered. The $50 \mathrm{~nm}$ thick layers of $\mathrm{Ag}$ or $\mathrm{Pt}$ were deposited at room temperature using a standard sputtering method at an argon pressure of 0.05 mbar (Sputter Coater SCD 050, Balzers, Germany). We additionally applied silver paste (Conrad Electronic GmbH, Germany) that was air dried at room temperature on top of the sputtered $\mathrm{Pt}$ electrodes in order to protect the thin electrodes from scratching during fatigue. Air dried silver paste did not adhere to the sputtered silver and fired pastes had to be used (Gwent Electronic Materials Ltd., UK, $400{ }^{\circ} \mathrm{C}$ ). Unfortunately, the fired silver paste strongly corroded the sputtered Pt electrodes, and this combination could not be used even though it would have provided a better comparability of the data between both sputtered electrode types.

The samples were fatigued under bipolar sinusoidal electric fields of amplitude $2 \mathrm{kV} / \mathrm{mm}\left(\approx 2 E_{c}\right)$ at $50 \mathrm{~Hz}$. After different cycle numbers, the fatigue treatment was interrupted and polarization $P$ and strain $S$ were measured in a separate setup. For the measurement of polarization, the samples were connected to a Sawyer-Tower circuit using a test capacitor of $15 \mu \mathrm{F}$. Strain was measured simultaneously by an optical displacement sensor. Polarization and strain hysteresis loops were measured as a function of a dc voltage superimposed on a weak ac voltage (amplitude of $5 \mathrm{~V} / \mathrm{mm}$ and frequency of $1 \mathrm{kHz}$ ). The dc voltage was incremented in steps of $100 \mathrm{~V} / \mathrm{mm}$ until the maximum or minimum fields of $\pm 2 \mathrm{kV} / \mathrm{mm}$ were reached. The dc step hold time was $1 \mathrm{~s} . d_{33}$ was measured from the optical sensor output using a lock-in amplifier (Stanford Research Systems 830). ${ }^{50}$

\section{B. PFM measurements}

After fatigue, the wedge-cut samples were prepared for the PFM measurements. ${ }^{51}$ They were embedded in epoxy resin, mounted at a tilt angle of about $10^{\circ}$ (Ref. 52) (see Fig. 1), ground, and polished. The advantage of preparing such a wedge is that the sample interior becomes accessible for PFM testing at all depths. PFM measurements are limited to a thin surface layer, the thickness of which depends on the experimental conditions and can vary from tens to a few hundred nanometers (Fig. 1). As the vertical component of the PFM signal is analyzed, the small angle furthermore permits measurement of the polarization distribution along the direction of the initially applied fatigue field direction to $98 \%\left[=\cos \left(10^{\circ}\right)\right]$. Due to macroscopic polarization and strain measurements after the fatigue procedure, all samples were in the poled state when embedded in the epoxy resin. The subsequent measurements confirmed that polishing did not alter this polarization state. ${ }^{53}$ The poling direction was consistently pointing into the sample (down in Fig. 1). Again diamond pastes were used as polishing agent $(15,6,3,1$, and $0.25 \mu \mathrm{m})$ with a final polishing step using Masterpolish® (Buehler). The samples were then etched in phosphoric acid for $20 \mathrm{~s}$ in order to display the grain boundaries.

The PFM measurements were performed using a commercial scanning probe microscope (Park Scientific Instruments Autoprobe CP-R). Details of the PFM imaging principles can be found elsewhere. ${ }^{54}$ A commercial Pt-coated silicon cantilever of spring constant $k=40 \mathrm{~N} / \mathrm{m}$ and a tip radius of less than $30 \mathrm{~nm}$ was used. The domains were displayed using an applied ac voltage of $3 \mathrm{~V}$ at a frequency of $12 \mathrm{kHz}$ which is far from the resonance frequency of the cantilever. The voltage of $3 \mathrm{~V}$ was chosen well beneath the limit where nucleation of new domains may occur underneath a PFM tip. ${ }^{55}$ The contact force between tip and sample was $25 \mathrm{nN}$. The domain structure was visualized in areas of $20 \times 20 \mu \mathrm{m}^{2}$. The line scanning frequency was $0.5 \mathrm{~Hz}$. To observe the switching behavior of the samples, a 10 $\times 10 \mu \mathrm{m}^{2}$ region within the $20 \times 20 \mu \mathrm{m}^{2}$ area was poled in steps by scanning the region with increasing dc voltages applied to the probing tip until the entire region was poled (poling scanning rate $=2 \mathrm{~Hz}$ ). After each poling step, the resulting domain configuration was imaged again and the amount of switched area was determined using image analysis software. These finite area switching experiments were performed in the wedge regions close to the bottom electrode where the ferroelectric layer was thinner than the average grain size. This allowed us to exclude the effect of grain boundaries aligned parallel to the electrode on domain imaging. In order to assess the effect of spontaneous backswitching after poling, some scans were performed under simultaneously applied poling $\mathrm{dc}$ and imaging ac voltages. The poling scanning rate was $0.5 \mathrm{~Hz}$ like for imaging. These experiments were performed on near electrode and bulk sections (Fig. 1). The local sample thickness was determined from the position of the tip on the wedge and the tilt angle.

\section{EXPERIMENTAL RESULTS}

\section{A. Fatigue behavior}

The general fatigue behavior of PIC 151 is illustrated by the macroscopic hysteresis loops for the two types of electrodes in Fig. 2. Ag electrodes yield a fairly good fatigue resistance compared to $\mathrm{Pt}$ electrodes on the same material. $11 \%$ loss of the initial polarization is observed within 3 $\times 10^{7}$ cycles. A similar fatigue cycle dependence of macroscopic strain as well as dielectric constant is observed. Samples with platinum electrodes showed a very broad range of degradation patterns. Some exhibited similar behavior 

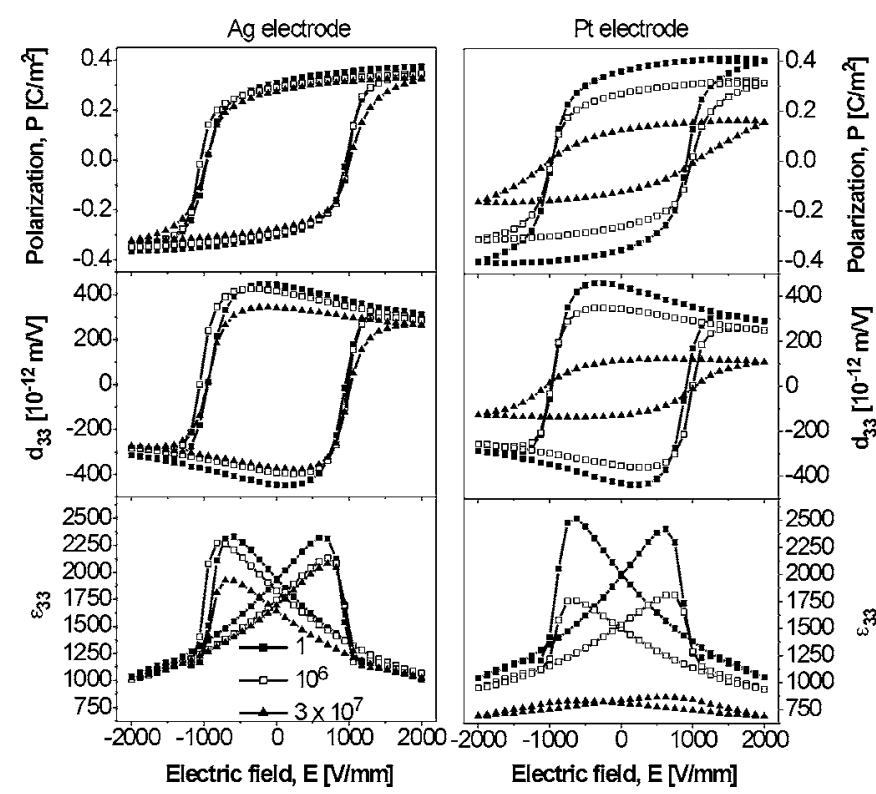

FIG. 2. Fatigue behavior of polarization, piezoelectric constant $d_{33}$, and dielectric constant $\varepsilon_{33}$ hysteresis loops for PIC 151 for two different electrode types. (Left) Sputtered and fired silver. (Right) Sputtered platinum.

such as silver, others a strong degradation of $2 P_{r}$ after $10^{5}$ cycles (Fig. 2, right column). The case of strongest reduction of $2 P_{r}$ is shown in Fig. 3 as a function of cycle number and compared to silver electrodes. As the macroscopic fatigue behavior consistently changed with the microscopic domain dynamics, we chose the most severely damaged case of Pt samples in order to best show the effects of fatigue. The patterns of other samples in between both lines were omitted for clarity.

\section{B. Silver electrodes}

In Fig. 4, the PFM phase and amplitude images are shown for a poled unfatigued, a semifatigued (3 $\times 10^{5}$ cycles), and a strongly fatigued $\left(3 \times 10^{7}\right) \mathrm{PZT} / \mathrm{Ag}$ sample. Note that all samples have been subjected to a high poling field during the macroscopic measurements prior to sample preparation for the PFM study. The virgin samples exhibit almost uniform PFM phase contrast and little variations in the PFM amplitude image, Fig. 4, which is an indication of complete domain switching in one direction. An increasing number of domains not aligned in poling direction

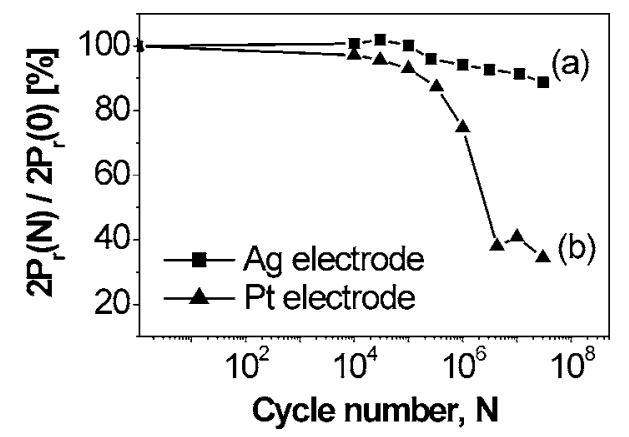

FIG. 3. Loss of switchable polarization during fatigue for two different electrodes on PZT: (a) Ag electrodes and (b) Pt electrodes exhibiting strong fatigue. (a)

(b)

(c)
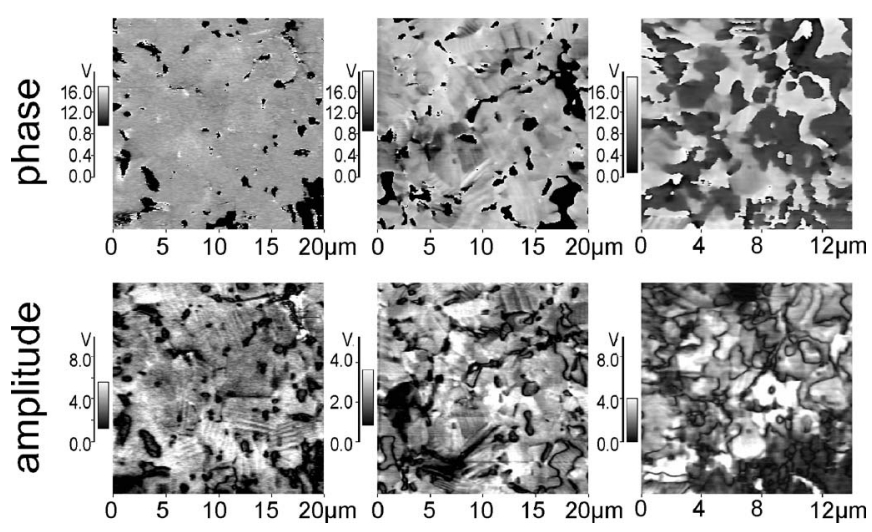

FIG. 4. Nonswitched polarization for the three fatigue stages: (a) nonfatigued, (b) $3 \times 10^{5}$ cycles, and (c) $3 \times 10^{7}$ cycles for PZT/Ag samples. The scanned area is $4 \mu \mathrm{m}$ thick PZT, wedge region (a), in Fig. 1.

appearing as dark regions in the phase images can be seen in the semifatigued and strongly fatigued samples. For the strongly fatigued sample, around $50 \%$ of the scanned area appears dark, varying somewhat from location to location.

The domains not aligned in poling direction can be switched by scanning the area with a voltage of $15 \mathrm{~V}$ dc applied to the PFM tip. Starting from the initial domain images in Fig. 4, finite area switching experiments were conducted for the unfatigued, semifatigued, and strongly fatigued samples under reversing voltage. In Fig. 5(a) the amount of switched area is displayed as a function of the applied dc voltage. The starting points at $0 \mathrm{~V}$ are different, because different amounts of domains remained nonreversed in the initial poling cycle after fatigue (dark areas in Fig. 4).
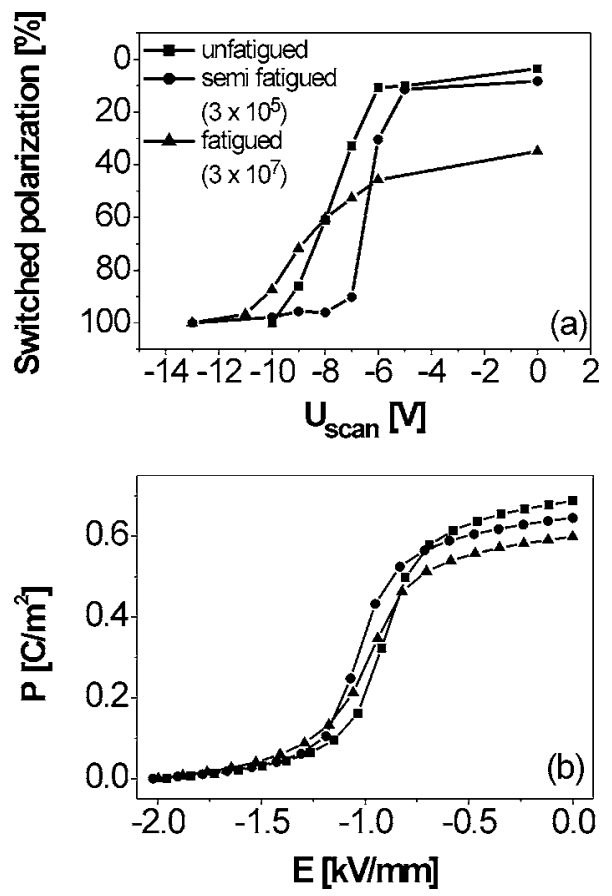

FIG. 5. Switched polarization from (a) finite area switching induced by the PFM tip in sections near the bottom electrode ( $4 \mu \mathrm{m}$ depth) and (b) macroscopic polarization hysteresis measurement for three different fatigue states for PZT/Ag sample $\left(0,3 \times 10^{5}\right.$ and $3 \times 10^{7}$ cycles $)$. 


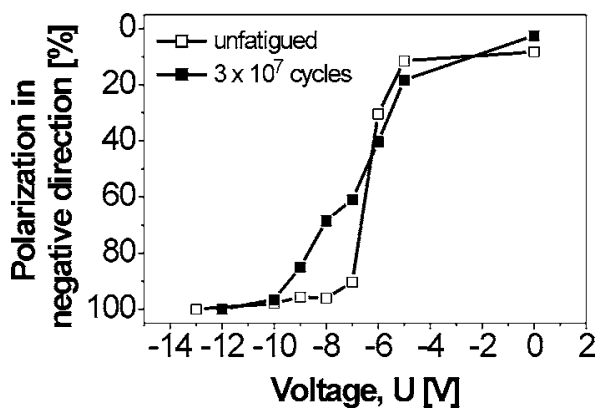

FIG. 6. Polarization switching by the PFM tip on a finite area in a thin sample region $(4 \mu \mathrm{m})$ for a PZT/Ag sample. The observed area had been poled in positive direction (by applying positive bias to the PFM tip). A nonfatigued sample is shown for comparison.

Figure 5(a) represents the switchable polarization on a local scale after PFM poling. The macroscopic switching data are shown in Fig. 5(b) for comparison.

Despite the large difference in probed volumes ${ }^{56}$ for the finite area switching with the PFM tip and the macroscopic hysteresis loops, the features for local and macroscopic measurements are similar. The distribution of $E_{c}$ is broader for the strongly fatigued samples in comparison with the unfatigued and semifatigued ones. The switched polarization is also lower for the fatigued sample. The dark areas in Fig. 4 for the fatigued samples can be switched by applying $15 \mathrm{~V}$ $\mathrm{dc}$ to the PFM tip. We repeated the finite area switching for the fatigued sample after complete poling with $15 \mathrm{~V}$ on the whole observed area. A comparison of the switched area between the fatigued and the unfatigued samples is shown in Fig. 6. Due to the renewed local poling of the fatigued sample, the finite area switching at $U=0 \mathrm{~V}$ starts from approximately the same poling state. The broader distribution of the coercive fields in the fatigued sample is still visible. The inflection points for both curves are at $-6.5 \mathrm{~V}$.

In regions of larger sample thickness (farther from the PZT/electrode border on the wedge), a strong backswitching effect is seen for fatigued samples (Fig. 7). At a thickness of approximately $300 \mu \mathrm{m}$, dark and bright domains are seen (a). During poling at $50 \mathrm{~V}$ dc plus $3 \mathrm{~V}$ ac, the phase image exhibits a bright contrast (b). After this poling treatment, the same phase image is seen as the initial one before poling (c). Nearly the entire area thus switches back into its original configuration.

(a)

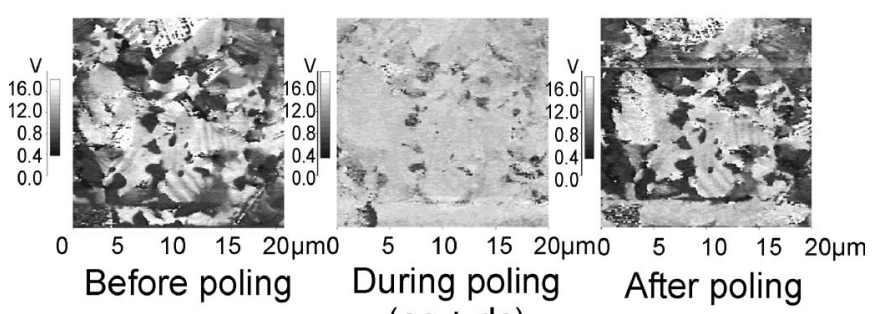

$(\mathrm{ac}+\mathrm{dc})$

FIG. 7. PFM phase images in a region of $300 \mu \mathrm{m}$ sample thickness: (a) before poling, (b) during poling with applied ac and dc voltages, and (c) after poling.

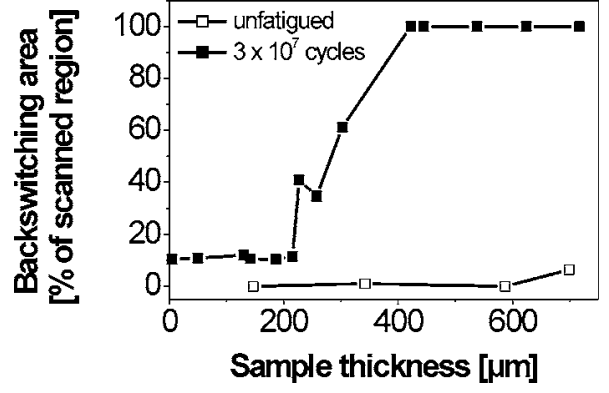

FIG. 8. Backswitched area for PZT/Ag (unfatigued and fatigued samples).

The amount of backswitched area after applying $50 \mathrm{~V}$ was compared for the unfatigued and the fatigued samples with $\mathrm{Ag}$ electrodes at different sample thicknesses (Fig. 8).

There is no significant backswitching effect in thin sections of the fatigued sample (up to $200 \mu \mathrm{m}$ ). It increases for larger sample thicknesses. In regions thicker than $400 \mu \mathrm{m}$ the poled area switches back completely. Backswitching is not due to sample preparation but to fatigue itself, because unfatigued samples show no backswitching whatsoever.

\section{Platinum electrodes}

In order to test the influence of a different electrode material of considerably different work function, all measurements were repeated for the PZT samples with sputtered Pt electrodes. In the top part of Fig. 9 the initial phase and amplitude images are seen in a region with less than one grain in thickness for a strongly fatigued $\left(3 \times 10^{7}\right.$ cycles $)$
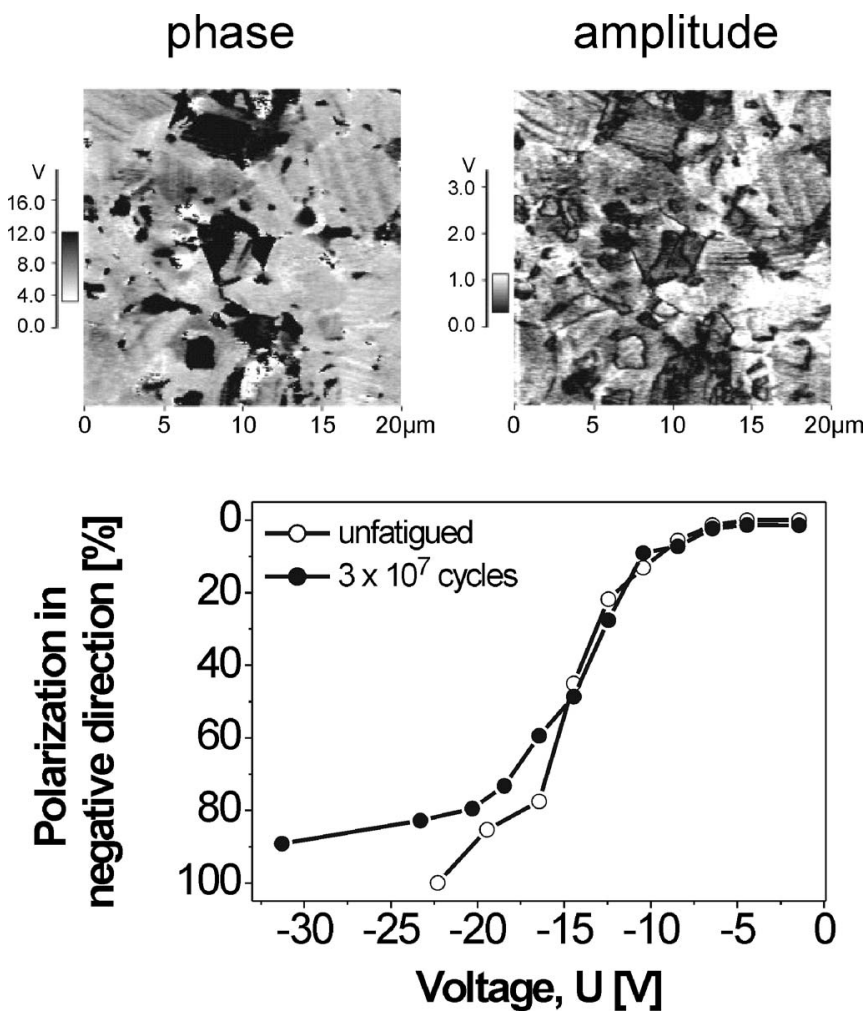

FIG. 9. (Top) Initial PFM image for the strongly fatigued $\left(3 \times 10^{7}\right.$ cycles $)$ PZT/Pt sample. (Bottom) Finite area switching for the unfatigued and strongly fatigued PZT/Pt samples after poling the observed area by the PFM tip. 


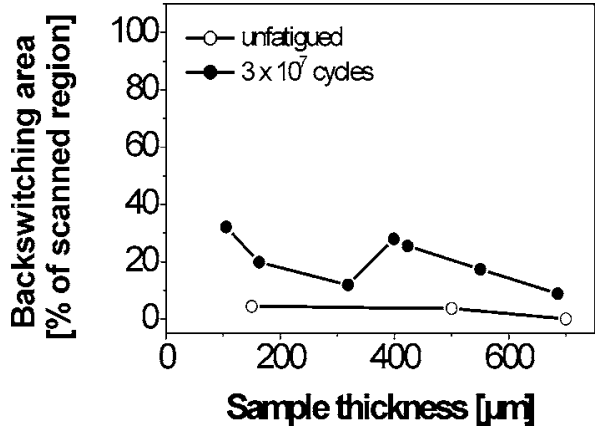

FIG. 10. Backswitched area for PZT/Pt (unfatigued and fatigued samples)

PZT/Pt sample. As in the case of the PZT/Ag sample, we see some nonpoled regions. In this case $24 \%$ of the area is not aligned in poling direction which is less than for Ag electrodes.

At the bottom of Fig. 9, finite area switching for the unfatigued PZT/Pt sample is compared with the strongly fatigued one. No big differences arise. The inflection points of the curves are at $-13 \mathrm{~V}$ which is twice as high as for the samples with Ag electrodes. Some samples with Pt electrodes do not exhibit as high field values at the inflection point as the one shown, while all PZT/Ag samples show practically identical field values. Backswitching after fatigue for thicker sample regions is not a strong effect for samples with Pt electrodes in contrast to the Ag electrodes (Fig. 10). The higher the coercive field observed in the thin near electrode region, the stronger the fatigue effect in these samples. Both effects are thus correlated.

For some Pt electrodes we observed many microcracks along the grain boundaries in the fatigued sample during finite area switching measurements. A three-dimensional (3D) image of the surface topography in the near electrode region is shown in Fig. 11. In the case of Ag electrodes, no microcracking was observed.

\section{DISCUSSION}

It has been suggested previously that a strong contribution to the degradation of switchable polarization in thin films arises due to charge entrapment and domain pinning in the near electrode region. ${ }^{15,18,57,58}$ As we showed previously, this is also true for bulk ferroelectrics. ${ }^{59,60}$ In this paper, we

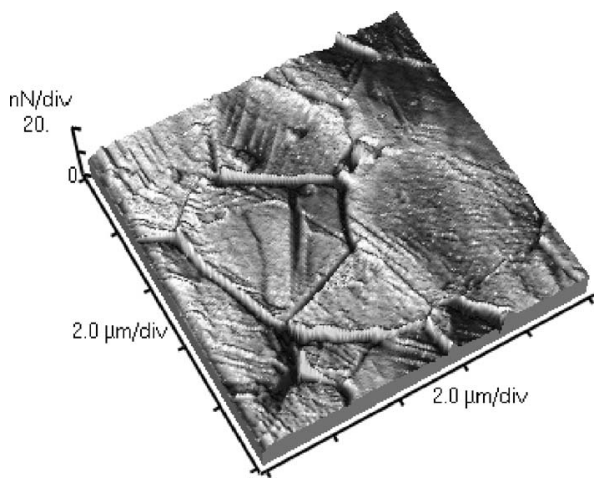

FIG. 11. 3D image of the surface topography (the scan area is 10 $\left.\times 10 \mu \mathrm{m}^{2}\right)$ from the thin region $(4 \mu \mathrm{m})$ in the fatigued PZT/Pt sample.

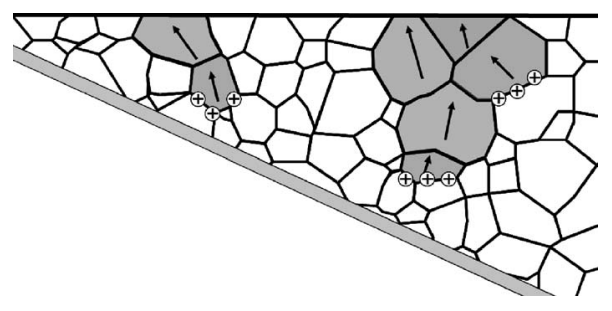

FIG. 12. Schematic drawing of a wedge shaped sample. Accumulation of space charge during fatigue in the bulk of the sample which is assumed to occur at grain boundaries.

chose samples with silver and platinum electrodes with the intent of having large differences in work functions of the metal electrodes and in order to test their relative effect on fatigue in a bulk material. ${ }^{1}$

Our measurements exhibit a significant difference in fatigue behavior between $\mathrm{Pt}$ and $\mathrm{Ag}$ electrodes. Certain samples with Pt electrodes exhibit a strong fatigue effect which is mainly due to microcracking in the near electrode region. Microcracking has been observed to occur in all cases where mechanical stresses are high such as in the vicinity of electrode edges from where microcrack clouds start growing. ${ }^{22}$ Here, the formation of microcracks is due to the broad distribution of local coercive fields underneath the electrodes for these particular electrodes, as displayed by the finite area switching analysis. A significant difference in local coercive fields $E_{c}$ leads to sequential switching of neighboring grains. When adjacent grains switch at different fields, mechanical stresses arise along the grain boundaries due to strain mismatch in between grains. ${ }^{61}$ Potentially, the first inversion cycle is capable of initiating first microcracks. If the immediate mechanical neighborhood of a grain changes due to such an initial microcrack, the switching characteristics of the adjacent grains further change and mechanical mismatch increasingly drives further microcrack formation. ${ }^{62}$ Subsequently, the low dielectric constant in the microcracks in the near electrode volume entails field screening in the sample bulk and thus shields this volume from further fatigue. The different fatigue behavior for PZT/Pt and PZT/Ag samples can be explained by the local distribution of coercive fields $E_{c}$ in the first layer of grains underneath the electrodes. The distribution of coercive fields itself is rather not affected by fatigue but is determined by the sputtering conditions of the electrodes. Apparently, the differences of the defect structure of the electrodes induced during sputtering are strong for Pt and not apparent for Ag. The latter shows a much more reproducible field value at the inflection point and a very reproducible fatigue pattern. This may also be due to better adhesion as a result of electrode firing in the silver case.

In the case of the PZT/Ag samples no field screening arises underneath the electrodes and fatigue takes place in the entire sample volume. In this case spontaneous backswitching develops after turning off the poling voltage. A simple microscopic mechanism that explains this backswitching effect is illustrated in Fig. 12 and described below.

We assume that fatigue leads to the gradual formation of charged grain boundaries (see, e.g., Shimada in Ref. 34) due 
(a)

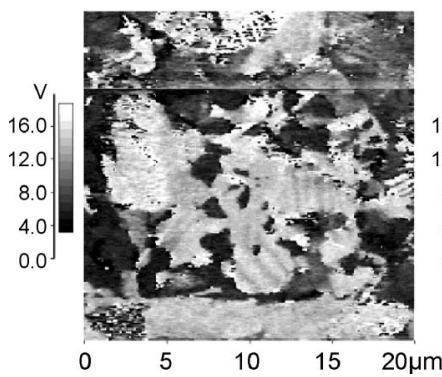

(b)

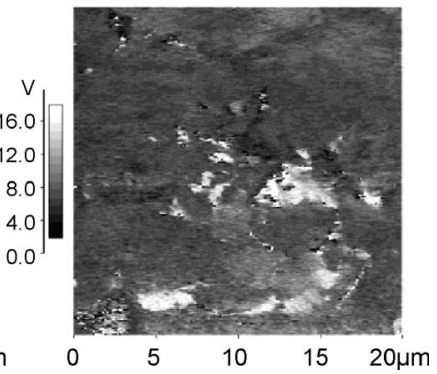

FIG. 13. Phase image of domains in PZT with Ag electrodes (fatigued sample) at a thickness of about $300 \mu \mathrm{m}$ after scanning with (a) $+50 \mathrm{~V}$ applied to PFM tip [see Fig. 6(c)] and (b) $-50 \mathrm{~V}$.

to the entrapment of free charges. As a result a grain assumes a preferred polarization orientation. This in turn changes the state of bound charges at the next grain boundaries and entails a preferred polarization orientation in the next layer of grains and so on leading to a columnar preferential polarization orientation in the grains throughout the sample thickness (gray grains in Fig. 12). This cascading is very similar to the common switching process during polarization reversal. ${ }^{50}$ The grains on the surface are still switchable in this state of the sample. The field generated by the PFM tip only acts locally at the surface and does not reach the grains lying deeper in the bulk. Polarization reversal thus only occurs locally. As soon as the external field is turned off, the charges and the grains deeper in the bulk switch the upper grains back. We tried to fully switch a region (sample thickness of $300 \mu \mathrm{m})$ in a fatigued sample with Ag electrodes at $+50 \mathrm{~V}$ applied to the PFM tip. The resulting PFM phase image measured after poling (Fig. 7) should display a completely bright contrast due to the polarization vector pointing down. But a domain pattern similar to the original one was observed after poling which indicates that backswitching occurred in some regions [Fig. 13(a)]. On the other hand, after poling by the tip under $-50 \mathrm{~V}$ bias, a homogeneous dark phase image is displayed [Fig. 13(b)]. Thus, only dark areas switch back and the polarization vector "up" is the obviously preferred orientation. The defects in the bulk must be of positive sign accordingly. With increasing sample thickness the charged grain boundaries become visible underneath the scanned region with a higher probability. This yields the observed thickness dependence of the backswitching area. The line of arguments on the localization of free charges at the grain boundaries is given here for simplicity. Even though there is no doubt about the existence of charges, there is yet no experimental evidence that they are located at the grain boundaries even though the finite mismatch of spontaneous polarization and the known differences in switching near the grain boundaries $^{35}$ makes this a likely location.

Surprisingly, the same polarity seems to be consistently found during electrical measurements of PZT for thin films. ${ }^{63,64}$ Do et al. ${ }^{63}$ investigated fatigue in PZT films (80 and $160 \mathrm{~nm}$ thick) by x-ray diffraction (XRD) reflection intensity measurements and Gruverman et al. ${ }^{64}$ studied the imprint behavior in small PZT capacitors. In both cases domain pinning arises in an orientation that corresponds to a posi-

tively charged back electrode which is the same orientation as in our experiment. The same polarity was also found without fatigue in samples that only possess a Pt back electrode and where no top electrode had ever been applied. ${ }^{65}$ Unfortunately, this knowledge still does not allow to discern whether the defects underneath the electrodes are really oxygen vacancies, ${ }^{23}$ protons, or electronic in nature. ${ }^{66}$ In the case of our samples, the sputtering conditions are moderately reducing at only 0.05 mbar which is far from common partial pressures during thin film preparation. As we observe a spread in coercive fields for platinum but not for silver, sputtering of the latter and subsequent annealing must afford the formation of a homogeneous stable interface to the ferroelectric. As there is no apparent difference in the preparation of different platinum electrodes, its deposition must be very sensitive to the sputtering conditions. A detailed investigation of such effects will require a well calibrated sputtering device. If a purely electronic interface scenario is responsible for the differences in fatigue behavior, the effective Schottky barrier must be significantly modified by different sputtering conditions in the case of platinum. Irrespective of these differences, the domain nucleation limited fatigue model by $\mathrm{Du}$ and Chen can explain the strong influence of the electrodes on the initial fatigue of our samples. ${ }^{67}$ At a later stage, effects deep in the sample arise for silver electrodes which do not directly influence domain nucleation. As the length scales in our study are three orders of magnitude larger than in the thin films, the same polarity of the free charges deep in the bulk can be accidental.

The quality and homogeneity of the electrode is also relevant for silver. For fired silver electrodes containing a glass binder on nonpolished surfaces of the ferroelectric, we previously observed that backswitching neither arises at a depth of $50 \mu \mathrm{m}$ nor at $100 \mu \mathrm{m}$ in bipolar fatigued bulk samples. ${ }^{68}$ We know that these samples degrade significantly in the electrode vicinity ${ }^{69}$ and the density of trapped charges deep in the bulk does not build up sufficiently during fatigue to induce backswitching. Overall the major fatigue effect in these samples also occurs underneath the electrodes due to the microscopic heterogeneity of the interface between the electrode and the ferroelectric. In the very thin sample region (less than one grain in thickness), a higher amount of lightly pinned domains was observed for the fatigued sample with Ag electrodes. The domains can be switched easily by applying a dc voltage of $15 \mathrm{~V}$ and are not pinned like it is found in thin films and assumed in some models to explain fatigue. ${ }^{19,70,71}$ Fatigue thus induces pinning of different strengths, but total clamping of the domains must be due to a very high defect density in thin films.

\section{CONCLUSION}

In the present study different fatigue behaviors for PZT bulk samples with different types of electrodes have been observed. A broad distribution of coercive fields is observed for PZT/Pt. In certain samples a large number of microcracks develop. These microcracks affect the internal electric field distribution, effectively screening the applied electric field and protecting the remainder of the sample bulk from further 
fatigue. In the case of PZT/Ag samples no microcracks have been observed and fatigue takes place in the entire volume of the sample. In this case, internal charged defects (presumably charged grain boundaries) of positive sign are generated which lead to the development of preferential polarization orientation (imprint).

\section{ACKNOWLEDGMENTS}

The authors are very much indebted to Thorsten Leist and Jonas Schäfer for the excellent help during sample preparation and the Deutsche Forschungsgemeinschaft for funding (SFB 595). The authors recognize the initiation of depth-dependent studies by Cyril Verdier and Yong Zhang and the idea of wedge shaped samples by Lukas Eng. Discussions with Jürgen Rödel are appreciated. The authors express their gratitude to Jacquie Hanson and Brian Rodriguez for help with PFM measurements.

${ }^{1}$ D. B. Fraser and J. R. Maldonado, J. Appl. Phys. 41, 2172 (1970).

${ }^{2}$ Q. Jiang, E. C. Subbarao, and L. E. Cross, J. Appl. Phys. 75, 7433 (1994).

${ }^{3}$ D. Wang, Y. Fotinich, and G. P. Carman, J. Appl. Phys. 83, 5342 (1998).

${ }^{4}$ H. Weitzing, G. A. Schneider, J. Steffens, M. Hammer, and M. J. Hoffmann, J. Eur. Ceram. Soc. 19, 1333 (1999).

${ }^{5}$ V. Bobnar, Z. Kutnjak, A. Levstik, J. Holc, M. Kosec, T. Hauke, R. Steinhausen, and H. Beige, J. Appl. Phys. 85, 622 (1999).

${ }^{6}$ M. Mitrovic, G. P. Carman, and F. K. Straub, Proc. SPIE 3992, 217 (2000).

${ }^{7}$ J. Nuffer, D. C. Lupascu, and J. Rödel, Acta Mater. 48, 3783 (2000).

${ }^{8}$ J. F. Scott, B. Pouligny, K. Dimmler, M. Parris, D. Butler, and S. Eaton, J. Appl. Phys. 62, 4510 (1987).

${ }^{9}$ J. F. Scott and B. Pouligny, J. Appl. Phys. 64, 1547 (1988).

${ }^{10}$ H. M. Duiker, P. D. Beale, J. F. Scott, C. A. Paz de Araujo, B. M. Melnick, J. D. Cuchiaro, and L. D. McMillan, J. Appl. Phys. 68, 5783 (1992).

${ }^{11}$ I. K. Yoo and S. B. Desu, Mater. Sci. Eng., B 13, 319 (1992).

${ }^{12}$ C. Brennan, Ferroelectrics 150, 199 (1993).

${ }^{13}$ C. J. Brennan, R. D. Parrella, and D. E. Larsen, Ferroelectrics 151, 33 (1994).

${ }^{14}$ W. L. Warren, D. Dimos, B. A. Tuttle, R. D. Nasby, and G. E. Pike, Appl. Phys. Lett. 65, 1018 (1994)

${ }^{15}$ P. K. Larsen, G. J. M. Dormans, D. J. Taylor, and P. J. van Veldhoven, J. Appl. Phys. 76, 2405 (1994).

${ }^{16}$ A. L. Kholkin, E. L. Colla, A. K. Tagantsev, D. V. Taylor, and N. Setter, Appl. Phys. Lett. 68, 2577 (1996).

${ }^{17}$ X. Du and I.-W. Chen, Appl. Phys. Lett. 72, 1923 (1998).

${ }^{18}$ X. Du and I.-W. Chen, J. Appl. Phys. 83, 7789 (1998).

${ }^{19}$ E. L. Colla, S. Hong, D. V. Taylor, A. K. Tagantsev, and N. Setter, Appl. Phys. Lett. 72, 2763 (1998).

${ }^{20} \mathrm{M}$. Grossmann, D. Bolten, O. Lohse, U. Boettger, R. Waser, and S. Tiedke, Appl. Phys. Lett. 77, 1894 (2000).

${ }^{21}$ B. G. Chae, C. H. Park, Y. S. Yang, and M. S. Jang, Appl. Phys. Lett. 75 2135 (1999).

${ }^{22}$ J. Nuffer, D. C. Lupascu, and J. Rödel, J. Eur. Ceram. Soc. 21, 1421 (2001).

${ }^{23}$ J. F. Scott and M. Dawber, Appl. Phys. Lett. 76, 3801 (2000).

${ }^{24}$ D. C. Lupascu and U. Rabe, Phys. Rev. Lett. 89, 187601 (2002).

${ }^{25}$ E. L. Colla, D. V. Taylor, A. K. Tagantsev, and N. Setter, Appl. Phys. Lett. 72, 2478 (1998)

${ }^{26}$ B. M. Melnick, J. F. Scott, C. A. Paz de Araujo, and L. D. McMillan, Ferroelectrics 135, 163 (1992).

${ }^{27}$ B. Li, F. Koch, X. J. Meng, J. G. Cheng, and J. H. Chu, Appl. Phys. Lett. 77, 898 (2000)

${ }^{28}$ I. K. Yoo and C. J. Kim, Integr. Ferroelectr. 11, 269 (1995).

${ }^{29}$ S. Takatani, H. Miki, K. Kushida-Abdelgahfar, and K. Torii, J. Appl. Phys. 85, 7784 (1999).

${ }^{30}$ M. Dawber and J. F. Scott, Jpn. J. Appl. Phys., Part 1 41, 6848 (2002).

${ }^{31}$ H. Kanaya, T. Iwamoto, Y. Takahagi, I. Kunishima, and S. Tanaka, Integr. Ferroelectr. 25, 235 (1999).

${ }^{32}$ A. Ruediger, O. F. Schirmer, A. K. Kadashchuk, and Yu. A. Skryshevskii, Europhys. Lett. 57, 592 (2002).
${ }^{33}$ M. Moert, G. Schindler, T. Mikolajick, N. Nagel, W. Hartner, C. Dehm, H. Kohlstedt, and R. Waser, Appl. Surf. Sci. 249, 23 (2005).

${ }^{34} \mathrm{H}$. Ishiwara, M. Okuyama, and Y. Arimoto, Ferroelectric Random Access Memories (Springer, Berlin, 2004).

${ }^{35}$ K. Franke, H. Huelz, and S. Seifert, Ferroelectr., Lett. Sect. 23, 1 (1997).

${ }^{36}$ A. K. Tagantsev, I. Stolichnov, E. L. Colla, and N. Setter, J. Appl. Phys. 90, 1387 (2001)

${ }^{37}$ M. Dawber, K. M. Rabe, and J. F. Scott, Rev. Mod. Phys. 77, 1083 (2005).

${ }^{38}$ W. Wu, K. H. Wong, C. L. Choy, and Y. H. Zhang, Appl. Phys. Lett. 77, 3441 (2000).

${ }^{39}$ C. A. Paz de Araujo, J. D. Cuchiaro, L. D. McMillan, M. C. Scott, and J. F. Scott, Nature (London) 374, 627 (1995).

${ }^{40}$ B. H. Park, B. S. Kang, S. D. Bu, T. W. Noh, J. Lee, and W. Jo, Nature (London) 401, 682 (1999).

${ }^{41}$ D. C. Lupascu, E. Aulbach, and J. Rödel, J. Appl. Phys. 93, 5551 (2003).

${ }^{42}$ C. Verdier, D. C. Lupascu, and J. Rödel, J. Eur. Ceram. Soc. 23, 1409 (2003).

${ }^{43}$ R. Ramesh et al., Appl. Phys. Lett. 61, 1537 (1992).

${ }^{44}$ I. K. Yoo, S. B. Desu, and J. Xing, Mater. Res. Soc. Symp. Proc. 310, 165 (1993).

${ }^{45}$ T. Nakamura, Y. Nakao, A. Kamisawa, and H. Takasu, Appl. Phys. Lett. 65, 1522 (1994).

${ }^{46}$ J. J. Lee, C. L. Thio, and S. B. Desu, J. Appl. Phys. 78, 5073 (1995).

${ }^{47}$ H. N. Al-Shareef, B. A. Tuttle, W. L. Warren, T. J. Headley, D. Dimos, J. A. Voigt, and R. D. Nasby, J. Appl. Phys. 79, 1013 (1996).

${ }^{48}$ J. F. Scott et al., J. Am. Ceram. Soc. 88, 1691 (2005).

${ }^{49}$ D. Ricinschi, M. Okuyama, and M. Shimiza, Appl. Phys. Lett. 81, 4040 (2002).

${ }^{50}$ Y. Zhang, D. C. Lupascu, E. Aulbach, I. Baturin, A. Bell, and J. Rödel, Acta Mater. 53, 2203 (2005).

${ }^{51} \mathrm{M}$. Alexe and A. Gruverman, Nanoscale Characterization of Ferroelectric Materials (Springer, Berlin, 2004).

${ }^{52}$ X. M. Lu, F. Schlaphof, S. Grafström, C. Loppacher, L. M. Eng, G. Suchaneck, and G. Gerlach, Appl. Phys. Lett. 81, 3215 (2002).

${ }^{53}$ Z. Wang, J. E. Blendell, G. S. White, and Q. Jiang, Smart Mater. Struct. 12, 217 (2003).

${ }^{54}$ A. Gruverman, O. Auciello, and H. Tokumoto, Annu. Rev. Mater. Sci. 28, 101 (1998)

${ }^{55}$ S. V. Kalinin, E. Karapetian, and M. Kachanov, Phys. Rev. B 70, 184101 (2004).

${ }^{56}$ S. V. Kalinin and D. A. Bonnell, in Nanoscale Characterization of Ferroelectric Materials, edited by M. Alexe and A. Gruverman (Springer, Berlin, 2004), pp. 1-43.

${ }^{57}$ M. Grossmann, O. Lohse, D. Bolten, U. Boettger, T. Schneller, and R. Waser, J. Appl. Phys. 92, 2680 (2002).

${ }^{58}$ M. Grossmann, O. Lohse, D. Bolten, U. Boettger, and R. Waser, J. Appl. Phys. 92, 2688 (2002).

${ }^{59}$ C. Verdier, F. D. Morrison, D. C. Lupascu, and J. F. Scott, J. Appl. Phys. 97, 024107 (2005)

${ }^{60}$ Y. Zhang, D. C. Lupascu, N. Balke, and J. Rödel, J. Phys. IV 128, 97 (2005).

${ }^{61}$ P. Chaplya and G. P. Carman, ASME Adaptive Structures and Materials Systems Symposium, Nashville, TN, November 1999, AD-Vol. 59, pp. 105-111.

${ }^{62}$ J. S. Liu, S. R. Zhang, L. S. Dai, and Y. Yuan, J. Appl. Phys. 97, 104102 (2005).

${ }^{63}$ D.-H. Do, P. E. Evans, E. D. Isaacs, D.-M. Kim, C.-B. Eom, and E. M. Dufresne, Nat. Mater. 3, 365 (2004).

${ }^{64}$ A. Gruverman, B. J. Rodriguez, A. I. Kingon, R. J. Nemanich, J. S. Cross, and M. Tsukada, Appl. Phys. Lett. 82, 3071 (2003).

${ }^{65}$ S. Dunn, J. Appl. Phys. 94, 5964 (2003).

${ }^{66}$ V. M. Fridkin, Ferroelectric Semiconductors (Consultants Bureau, New York, 1980).

${ }^{67}$ X. Du and I.-W. Chen, Mater. Res. Soc. Symp. Proc. 493, 311 (1998).

${ }^{68}$ V. V. Shvartsman, A. L. Kholkin, C. Verdier, and D. C. Lupascu, J. Appl. Phys. 98, 094109 (2005).

${ }^{69}$ J. Nuffer, D. C. Lupascu, A. Glazounov, H.-J. Kleebe, and J. Rödel, J. Eur. Ceram. Soc. 22, 2133 (2002).

${ }^{70}$ A. Gruverman, O. Auciello, and H. Tokumoto, Appl. Phys. Lett. 69, 3191 (1996).

${ }^{71}$ V. Ya. Shur, E. L. Rumyantsev, E. V. Nikolaeva, and E. I. Shishkin, and I. S. Baturin, Phys. Solid State 44, 2151 (2002) [Fiz. Tverd. Tela (S.Peterburg) 44, 2055 (2002)]. 